\title{
Carnets
}

Revue électronique d'études françaises de l'APEF

Deuxième série - $12 \mid 2018$

Théorie Mimétique et Études Littéraires

\section{Dandysme, crise du modèle épique et crise sacrificielle : Psycho(patho)logie du héros fin-de- siècle dans sa volonté d'être unique}

Marie Kawthar Daouda

\section{OpenEdition}

Journals

Édition électronique

URL : http://journals.openedition.org/carnets/2499

DOI : $10.4000 /$ carnets. 2499

ISSN : 1646-7698

Éditeur

APEF

Référence électronique

Marie Kawthar Daouda, «Dandysme, crise du modèle épique et crise sacrificielle : Psycho(patho)logie du héros fin-de-siècle dans sa volonté d'être unique », Carnets [En ligne], Deuxième série - 12 | 2018, mis en ligne le 23 janvier 2018, consulté le 05 mai 2019. URL : http://journals.openedition.org/ carnets/2499; DOI : 10.4000/carnets.2499

Ce document a été généré automatiquement le 5 mai 2019.

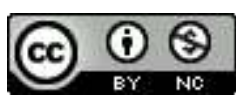

Carnets est mis à disposition selon les termes de la licence Creative Commons - Atribution - Pas d'utilisation commerciale 4.0 International. 


\title{
Dandysme, crise du modèle épique et crise sacrificielle : Psycho (patho)logie du héros fin-de-siècle dans sa volonté d'être unique
}

\author{
Marie Kawthar Daouda
}

« Le dandysme est, ou devrait être, synonyme de générosité, de dépouillement, d'élan vers la sainteté."

(Gabriel Matzneff, 1994 : 75)

1 René Girard illustre la théorie du désir mimétique en s'appuyant sur des exemples aussi divers que l'ÆEdipe roi de Sophocle ou l'anorexie contemporaine ${ }^{1}$. Le système du désir triangulaire trouve, certes, sa source chez Cervantès dans les premières pages de Mensonge romantique et vérité romanesque, mais c'est particulièrement sur une partie du $\mathrm{XIX}^{\mathrm{e}}$ siècle élargi, de la Restauration de Stendhal à la Belle Époque de Proust, que Girard puise ses exemples français. Depuis la Révolution, chaque changement de régime semble décapiter la France, l'amputer de ses illusions. Le roman réaliste participe à ce démantèlement du rêve napoléonien en désagrégeant l'épopée. Dans son analyse d'À la recherche du temps perdu, René Girard souligne l'absence de différence entre le champ de bataille et le salon, l'émulation épique se dissolvant dans le snobisme mondain. Joris-Karl Huysmans est né en 1848, Jean Lorrain en 1855. Ils ont respectivement 22 et 15 ans lors de la défaite de Napoléon III à Sedan. Lorrain est de vingt ans l'âné de Proust, qui le provoque en duel le 6 février 1897. À rebours de Huysmans, paru en 1884, et Monsieur de Phocas de Jean Lorrain paru en 1901, érigent les dernières heures avant la Belle-Époque en « fin de siècle » se percevant essentiellement comme une fin d'empire, où l'épopée n'a plus cours. La période décadente montre la crise sacrificielle sous son aspect particulier de crise du modèle épique. 
2 À rebours et Monsieur de Phocas tiennent du catalogue d'étrangetés esthétiques où « le beau est toujours bizarre» (Baudelaire, 1868: t. 2: 216). Jean des Esseintes, le héros de Huysmans, et Jean de Fréneuse, celui de Lorrain, derniers héritiers du dandysme, affichent par leur goût de l'objet précieux et par leurs fantaisies esthétiques un souverain mépris du monde. S'ils ont une réputation sulfureuse, ces personnages ont davantage une attitude de stylites que de débauchés. Selon Baudelaire, le dandy est la figure intermédiaire entre décadence et renaissance. Il "apparaît surtout aux époques transitoires. (...) Le dandysme est le dernier éclat d'héroïsme dans les décadences.» (Baudelaire, 1954 : 908) Renonçant au bonheur, des Esseintes et Fréneuse s'entourent de beauté, se font eux-mêmes œuvres d'art et entretiennent ainsi « une espèce de culte de soi-même qui peut survivre à la recherche du bonheur à trouver dans autrui, (...) qui peut survivre même à tout ce qu'on appelle les illusions.» (Baudelaire, 1885: 93) Collectionneurs, des Esseintes et Fréneuse semblent ne s'entourer de beauté que pour confirmer leur permanente insatisfaction. Le goût de l'idéal, en condamnant à la déception, enclenche une ascèse à rebours. Le spleen se fait symptôme d'une insatisfaction irrévocable qui pousse à l'immobilité ou à une quête interminable dans laquelle s'exténuent les derniers élans de l'épopée.

3 L'héroïsme de des Esseintes et de Fréneuse s'écrit en opposition au modèle épique du héros guerrier - Achille, voyageur - Ulysse, fondateur - Énée, et de la synthèse constituée par le mythe napoléonien. Le héros fin-de-siècle est à la fois fuyant et statique, et volontiers destructeur ou auto-destructeur. À rebours développe le cas clinique de des Esseintes quittant Paris et créant, à Fontenay-sous-Bois, une thébaïde où il espère que l'enfermement solitaire avec des œuvres d'une beauté singulière le guérira de la laideur du monde. Il y rassemble des livres rares, des tableaux étranges, y développe un orgue à parfums, collectionne des fleurs aux formes suggestives et au parfum étouffant. La salle à manger figure une cabine de navire ; la chambre à coucher imite l'austérité d'une cellule monastique. Loin de guérir des Esseintes, la solitude ne fait qu'exacerber sa neurasthénie et son hypersensibilité, et l'intervention d'un médecin met un terme à l'expérience : des Esseintes doit regagner le monde sous peine de mourir de son désir d'être unique.

4 Monsieur de Phocas reprend la même présentation du personnage comme cas clinique. Le duc Jean de Fréneuse confie son journal intime au narrateur au nom de leur amour pour les pierres rares, avant de partir pour l'Orient sous le pseudonyme de monsieur de Phocas. Dans ce journal, le duc détaille l'évolution du mal qui le tue : il est obsédé par une lueur verte qu'il guette dans les joyaux et dans les yeux des portraits, jusqu'à la folie et jusqu'au crime.

5 La théorie mimétique élaborée par René Girard permet de voir dans le désir maladif du bel objet un désir pathologique d'être unique. Ce désir est le moteur et le symptôme de la crise sacrificielle que connaît la France à la fin du XIXe siècle. Des Esseintes et Fréneuse sont deux personnages isolés, qui revendiquent leur radicale différence. Pourtant, cette maladie de l'unicité ne fait que souligner l'impossibilité de se concevoir comme individualité désirante. En l'absence de médiateur, le duc des Esseintes est physiquement exténué par ses désirs de plus en plus aberrants. Quant au duc de Fréneuse, il est soumis à l'influence du premier médiateur venu. Son journal s'étend sur près de dix ans, mais le cœur de l'intrigue a lieu dans la dernière année, entre 1898 et 1899, où le duc de Fréneuse est tiraillé entre deux médiateurs, l'inquiétant peintre Claudius Ethal et le nietzschéen Thomas Welcôme. 
6 Pour étudier le lien entre crise sacrificielle et crise du modèle épique, nous lirons À rebours et Monsieur de Phocas à travers les trois étapes identifiées par René Girard dans le récit mythique, la crise, le sacrifice puis la sanctification. Dans un premier temps, nous considérerons la crise comme tension entre apparent désir d'unicité et réel désir mimétique dans le roman postromantique. L'illusion de l'originalité, poussée jusqu'au désir destructeur ou autodestructeur, sera ensuite mise en relation avec la pensée politique du sacrifice que développe René Girard dans Le Bouc émissaire (1982) et dans Je vois Satan tomber comme l'éclair (1999). L'étude des dynamiques politiques sacrificielles conduira à voir en des Esseintes et Fréneuse, comme idolâtres d'art, les emblèmes d'une société où Dieu est mort et dont ils sont à la fois, pour reprendre la formule de Baudelaire «la victime et le bourreau».

\section{I - Originalité et folie : le roman comme cas clinique}

\section{Jusqu'au ridicule}

7 L'idéal poursuivi par le dandy fait de lui un adepte de l'acribie esthétique. Dans un projet de préface pour Les Fleurs du mal, Baudelaire envisage d'écrire « [c]omment, par une série d'efforts déterminée, l'artiste peut s'élever à une originalité proportionnelle» (Baudelaire, 1908 : 17). C'est le même objectif d'ascèse triplement répété à travers le vœu d'être « un héros et un saint » (Baudelaire, $1954: 1219-1222)$. Le héros et le saint ont pour point commun l'acceptation d'une mission ou d'une vocation. L'un comme l'autre rejettent la norme, évoluent dans l'excessif, l'extrême et l'extraordinaire. Dans la crise religieuse et la crise épique de la fin du XIX siècle, l'héroïsme et la sainteté semblent relever davantage de la névrose que de la noblesse. À rebours et Monsieur de Phocas sont des études cliniques. Selon Maupassant, À rebours pourrait s'intituler « histoire d'une névrose ». La vie de des Esseintes jusqu'à l'expérience de la thébaïde tient en quelques pages, ce qui souligne l'importance moindre de la matière narrative du roman à comparaison de l'enfermement qui lui succède, où l'action est abolie au profit de la quête de la sensation rare. Dans Monsieur de Phocas, le narrateur s'efface pour mettre sous les yeux du lecteur le journal où le duc de Fréneuse étudie sa propre névrose et se décrit comme un monstre seul contre tous : «Tous et toutes sentent en moi un être hors nature, un automate galvanisé de convoitises, mais un automate, c'est-à-dire un mort, et je leur fais peur avec mes yeux de cadavre " (Lorrain, 1901 : 288). La mobilité de la quête épique est ainsi supplantée par la rigidité cadavérique, comme le regard héroïque à l'horizon est remplacé par le regard centré sur soi, associé grâce au naturalisme à l'incursion du médical dans le littéraire. Le bromure et la valériane sont mentionnés à plusieurs reprises comme faisant partie du traitement de Fréneuse. À la fin d'À rebours, le titre est justifié d'une façon quasi-rabelaisienne puisque les dérangements gastriques de des Esseintes l'empêchent de s'alimenter autrement que par lavements - à rebours du tube digestif. Le désir pathologique d'originalité tourne à la folie risible, soulignée par le "burlesque " (Genette, 1982 : 29-30) du style d'écriture quand des Esseintes se réjouit :

Quel définitif débarras de la lassitude qui découle toujours du choix forcément restreint des mets ! Quelle énergique protestation contre le bas péché de la gourmandise ! enfin quelle décisive insulte jetée à la face de cette vieille nature dont les uniformes exigences seraient pour jamais éteintes! (Huysmans, 1922 : 274)

Ni À rebours ni Monsieur de Phocas ne tiendraient sans la possibilité d'une lecture au «second degré ». Des Esseintes est un raffiné amateur de sensations rares, mais sa plus 
grande joie vient du brutal arrachage d'une molaire cariée chez un dentiste du peuple. Le duc de Fréneuse passe pour un roué, mais il s'évanouit après avoir fumé du kief dans une soirée organisée par Ethal. Les incohérences des personnages, loin d'amoindrir le texte, lui assurent sa cohérence aux frais de la splendeur du héros romantique. Cette dimension burlesque, constitutive du héros fin-de-race, fut d'emblée perçue comme telle par Maupassant. Il décrit À rebours comme « extravagant et désopilant » et poursuit :

Jean des Esseintes, ayant touché à tous les plaisirs, à toutes les choses réputées charmantes, à tous les arts, à tous les goûts, trouvant insipide la vie, odieuses les heures monotones et semblables, se fabrique, à force d'imagination et de fantaisie, une existence absolument factice, absolument cocasse, vraiment à rebours de tout ce qu'on fait ordinairement (Maupassant, $1884: 1$ ).

9 L'ennui du héros aux désirs désorientés est indissociable de l'humour avec lequel il est présenté. Ce procédé rappelle la "prodigieuse bouffonnerie » (Girard, $2010: 294)$ relevée par Girard dans Le Sous-sol de Dostoievski. Des Esseintes et Fréneuse ne cessent de dire : « Moi, je suis seul, et eux, ils sont tous » (Girard, $2010: 294)$, les deux héros veulent « exprimer l'orgueil et la souffrance d'être unique » (Girard, $2010: 294$ ), mais la théorie mimétique montre combien ce désir d'être unique par le goût de l'objet rare n'est qu'une forme exacerbée de désir imitatif.

Héros fin-de-race, des Esseintes et Fréneuse ont un air de famille et semblent les deux derniers rejetons de lignées apparentées. Le terme de héros fin-de-race porte à l'illusion romantique de l'originalité un coup fatal : loin d'être unique, le personnage est déjà une copie grevée d'hérédité. Des Esseintes et Fréneuse héritent de leur mère respective leur mélancolie et leur sensibilité nerveuse. Les maux du corps et de l'esprit se reçoivent comme autant de tares et de malédictions. Si Monsieur de Phocas est une redite d'À rebours, À rebours arrive déjà comme l'épilogue du dandysme, tombé à l'excentricité, et manifeste la chute de l'originalité dans le domaine public. Monsieur de Phocas, par ses nombreuses allusions à des personnes réelles du Paris fin-de-siècle, est aussi un roman à clefs. Centré qu'il soit sur le personnage de Fréneuse, le roman compose autour de lui une "danse macabre » mondaine. Madeleine Deslandes ${ }^{2}$ et Robert de Montesquiou, l'un des modèles de Fréneuse, se trouvent ainsi épinglés au rang des originalités vaines: "La baronne Desrodes est la femme des grenouilles, comme le comte de Montesquiou est l'homme des chauves-souris, et tous deux croient révolutionner le monde... ô pauvretés! ô mesquineries! ô vanités!» (Lorrain, 1901: 96). Chaque personnage arborant son originalité comme un stigmate désormais insignifiant, aucun ne peut amorcer une nouvelle quête ni enclencher de crise sacrificielle. Reste à rire de ces ridicules et, à défaut d'écrire une quête épique, à écrire son impossibilité. C'est ainsi que l'idéal de fondation motivant la quête du héros épique est supplanté par l'idole, l'objet rare adoré par l'esthète.

\section{L'idolâtrie de l'art}

Des Esseintes et Fréneuse ne sont pleinement compréhensibles que comme redites. Lorsque le narrateur découvre le duc de Fréneuse, son impression est celle d'un déjà-vu :

Étroitement moulé dans un complet de drap vert myrthe, cravaté très haut d'une soie vert pâle et comme sablée d'or, $M$. de Phocas était un frêle et long jeune homme de vingt-huit ans à peine, à la face exsangue et extraordinairement vieille, sous des cheveux bruns crespelés et courts. Ce profil précis et fin, la raideur voulue de ce long corps fluet, l'arabesque (si je puis m'exprimer ainsi), l'arabesque 
tourmentée de cette ligne et de cette élégance, j'avais déjà vu tout cela quelque part. (Lorrain, $1901: 2$ ) impression de déjà-vu, ou plutôt de déjà-lu, est liée à la construction du type de l'esthète. Apparemment solitaire et isolé, l'esthète comme idolâtre d'art est au contraire relié à un réseau d'influences et d'affinités plus ou moins explicites. La prétention à la solitude, poussée jusqu'à la névrose, rend plus flagrantes les influences auxquelles il est soumis. La quête du bel objet rare prenant la place de la quête épique est quichottesque dans le sens où des Esseintes comme Fréneuse cherchent moins ce qui est beau que ce dont ils sont les seuls à percevoir la beauté. Il suffit qu'un poète ou un artiste soit aimé de la foule pour que des Esseintes lui retire son admiration :

L'œuvre d'art qui ne demeure pas indifférente aux faux artistes, qui n'est point contestée par les sots, qui ne se contente pas de susciter l'enthousiasme de quelques-uns, devient, elle aussi, par cela même, pour les initiés, polluée, banale, presque repoussante.

Cette promiscuité dans l'admiration était d'ailleurs l'un des plus grands chagrins de sa vie; d'incompréhensibles succès lui avaient à jamais gâté des tableaux et des livres jadis chers ; devant l'approbation des suffrages, il finissait par leur découvrir d'imperceptibles tares, et il les rejetait, se demandant si son flair ne s'épointait pas, ne se dupait point. (Huysmans, $1922: 130-131$ )

Ce flair est moins celui du collectionneur que du critique, guidé par ce qu'il y a de plus intérieur mais de plus imitable, la sensation décrite. Chez Huysmans comme chez Lorrain, la critique d'art concurrence la narration. Outre à pouvoir se lire comme un catalogue du musée idéal, les ekphraseis peuvent reprendre ou annoncer un écrit sur l'art. Huysmans romancier dans À rebours et critique d'art dans L'Art moderne poursuit le même éloge d'Odilon Redon. Un an après la parution d'À rebours, «Le Nouvel album d'Odilon Redon » conserve la même ligne directrice : Redon est à la fois inclassable, unique, et rattaché à un réseau complexe d'influences poétiques où figurent, en tête, Baudelaire et Poe. Quand l'auteur fait ainsi l'éloge des artistes qui l'enthousiasment, il insère artiste et esthète dans un réseau d'admirations. La solitude de des Esseintes n'est qu'apparente : il est environné d'artistes choisis dont certains, Baudelaire, Odilon Redon, Gustave Moreau, Stéphane Mallarmé, Barbey d'Aurevilly, font partie du cercle d'amis ou de relations de Huysmans. Loin de manifester la singularité du personnage ou celle de l'artiste admiré, ce réseau de complicités esthétiques leur confère au contraire une légitimité, liée à l'autorité de figures de maîtres.

Chez Lorrain, la critique en vient même à se taire devant la citation, en témoignent les paragraphes d'un conte de Charles Vellay, "Contes au bord de la mer ", publié en juin 1897. La hantise de Fréneuse, sa fascination pour la lueur glauque de certains regards, est la pathologie distinctive dont il porte le stigmate parmi les héros fin-de-race. Pourtant, cette singularité s'appuie sur un emprunt au conte de Vellay, sur un travail de citation et de réécriture. Le caractère imitatif du désir se double du caractère imitatif de l'écriture de Lorrain. Comme chez Huysmans, le critique d'art a partie liée avec le romancier. Dans une chronique parue dans Le Journal du 30 juin 1897, Lorrain évoque la découverte de ce conte de Vellay, lu par une jeune femme : «Et il disait des choses merveilleuses, ce conte : Au bord de la mer, qu'elle lisait, de sa voix chantante et grave, dans une revue de jeunes, le Mercure de France, je crois » (Lorrain, 2007 : 335). Le conte de Charles Vellay paraît dans une livraison du Mercure de France où figurent également quatre poèmes de Lorrain. La feinte ignorance ne rend que plus tangible le réseau artistique où s'insère l'œuvre rare. 
Derrière le Mercure de France, ce sont les amitiés - ou inimitiés - parisiennes de Lorrain qui s'esquissent, Rachilde, Marcel Schwob, Remy de Gourmont. La lectrice, surtout, est d'autant plus présente que son nom est tu : il s'agit de Jeanne Jacquemin, «peintresse de rêve et de mystère » (Lorrain, 2007 : 35). Si l'amitié entre Lorrain et Jacquemin s'achève par un procès fracassant, fatal à l'auteur de Monsieur de Phocas, ils sont néanmoins unis par Henry Kistemaekers dans la dédicace de la nouvelle «L'Heure du sang » : " Pour Jean Lorrain et Jeanne Jacquemin, dans leur communion d'art » (Kistemaekers, 1897 : 63). Chez Redon, Vellay, Jacquemin et Lorrain, la fascination pour les yeux suscite la même fécondité artistique. Il s'agit bien d'une communion, et des Esseintes et Fréneuse, en se faisant l'écho des sympathies de l'auteur et du culte esthétique dont il est le héraut, sont à la fois des initiés et des initiateurs.

Le caractère ésotérique, donc initiatique, de références artistiques dont le héros isolé se fait hiérophante et pédagogue est une fissure dans sa tour d'ivoire ou de verre. L'enfermement apparent du héros est traversé par la voix de l'auteur qui lui octroie son propre rôle d'initiateur. Ainsi, le chapitre III d'À rebours cumule les références à la latinité tardive, comme pour garder les barbares hors du temple, selon le leitmotiv d'À rebours. Cette opacité a pourtant pour effet d'enfermer entre eux les initiés. Cela est d'autant plus manifeste dans Monsieur de Phocas, où les allusions vagues sont moins présentes que d'amples citations. Le lieu d'isolation choisie, que ce soit la thébaïde de des Esseintes ou le journal intime de Fréneuse, devient tout à coup un cercle de réunions esthétiques.

16 Le désir de solitude cache un appel plus ou moins patent de l'élitisme. La volonté pathologique d'être unique par le goût de la rareté est le symptôme d'un désir d'appartenance. Quel que soit l'objet sur lequel elle se fixe, la quête de la sensation rare pour des Esseintes, de la lueur verte pour Fréneuse, les rendent littéralement malades de désir. Des Esseintes et Fréneuse ressemblent autant à Emma Bovary qu'à saint Antoine. Huysmans et Lorrain sont bien héritiers de Flaubert, à la fois conscients de la vanité au cœur du désir mimétique et du caractère mimétique de ce vide existentiel. «Le snob désire le néant » (Girard, 2010 : 249) ; justement, des Esseintes et Fréneuse ne désirent rien d'autre que le désir lui-même. Si le désir des héros est si violemment transporté d'un objet vers l'autre, c'est parce qu'il n'y a plus de médiateur.

\section{II - Violence sacrificielle et lucidité romanesque}

17 La volonté pathologique d'être unique souligne le désir d'appartenance. Cette situation présentée comme singulière relève du constat anthropologique, et offre une clé d'interprétation à la violence de cette période indécise qui précède la Belle Époque. À la différence du héros romantique qui nie le sien et le dissimule, le héros fin-de-siècle se cherche un médiateur, dont l'absence déclenche la crise du modèle épique.

Nos héros sont certes risibles par leur fanfaronnade quichottesque, mais ni À rebours ni Monsieur de Phocas ne cachent que la solitude du héros sans médiateur entraîne un vide existentiel. Le ridicule de des Esseintes cohabite avec sa détresse :

Une fois de plus, cette solitude si ardemment enviée et enfin acquise, avait abouti à une détresse affreuse ; ce silence qui lui était autrefois apparu comme une compensation des sottises écoutées pendant des ans, lui pesait maintenant d'un poids insoutenable. Un matin, il s'était réveillé, agité ainsi qu'un prisonnier mis en cellule ; ses lèvres énervées remuaient pour articuler des sons, des larmes lui 
montaient aux yeux, il étouffait de même qu'un homme qui aurait sangloté pendant des heures (Huysmans, $1922: 164$ ).

l'héritage baudelairien. René Girard identifie Satan au « mimétisme conflictuel » (Girard 1999 : 69). Dans la solitude, comme l'illustre chez Flaubert La Tentation de saint Antoine, le désir mimétique ne disparaît pas mais s'exaspère : il porte de manière successive sur tous les objets absents, du fait même de leur absence. L'impuissance sexuelle de Des Esseintes et de Fréneuse illustre le désir égaré, fragmenté. Des Esseintes organise le célèbre dîner en noir pour marquer la mort de sa virilité ; le duc de Fréneuse, en visitant les lieux de son enfance, regrette l'intensité des désirs qui l'habitaient alors - ces héros sont à peine trentenaires. En réalité, chez des Esseintes comme chez Fréneuse, le héros est trop conscient de désirer le médiateur, de désirer être le médiateur, pour désirer encore l'objet. Des Esseintes est un médiateur raté. Le chapitre VI le montre échouant dans son dessein de changer un jeune ouvrier en criminel en attisant son désir mimétique. L'épisode de la tartine, au chapitre XIII, révèle également la faillite du héros comme médiateur. Alors que tout l'écœure et qu'aucun mets ne tente plus son appétit, des Esseintes est pris d'un désir violent pour une tartine que mange un enfant. Lorsqu'il s'en fait faire une toute pareille, son désir tourne à l'écoeurement. La crise qu'engendre le héros comme médiateur est dérisoire : c'est la bataille des garçons s'arrachant la tartine qu'il leur a fait jeter. La pulsion n'est pas satisfaite par l'assouvissement du désir, mais par la communication de ce désir. Le caractère statique, inefficace, de des Esseintes comme héros souligne paradoxalement la médiation comme cœur de la fonction héroïque.

21 Le rapport entre le duc de Fréneuse et ses médiateurs est encore plus explicitement romanesque. Claudius Ethal fait désirer la guérison à Fréneuse, puis disparaît pour chercher l'objet de son propre désir, la « Merveille de Leyde », une poupée de cire. Après son soudain rapprochement, l'éloignement plus soudain encore du médiateur suscite la manifestation du désir :

(...) et mon Claudius ne revient pas.

Quelle place il a prise dans ma vie, comme il me manque ! Sa présence m'est devenue tellement nécessaire que, depuis son absence, comme une faim me creuse et me tenaille l'être. C'est une sensation de faim absolument, et en même temps j'étouffe et je suffoque. Et pourtant, je le sens, je crains et je hais cet Anglais de malheur (Lorrain, 1901 : 104).

Ce désir est tel que le médiateur cesse d'en être un : Ethal est déçu de voir que sa poupée de cire laisse Fréneuse indifférent (Lorrain, 1901: 130-131). Le duc est lucide sur l'inextricable mixte d'amour et de haine suscité par le médiateur. L'initiation homosociale au désir, analysée par Girard dans le triangle de la médiation interne, se développe en initiation homoérotique dans l'attirance de Fréneuse pour Ethal puis pour Thomas Welcôme. Quand Ethal cherche à se poser en médiateur, sa stratégie échoue et il 
ne peut faire désirer la « Merveille de Leyde » à Fréneuse ; en revanche, l'impatience de Fréneuse désirant retrouver Ethal s'exprime dans des termes similaires à ceux où apparait la fascination d'Ethal pour Welcôme ${ }^{3}$.

Je n'avais jamais vu Claudius si ému.

Sir Thomas Welcôme s'inclinait, très froid. C'était un très beau cavalier avec une figure douce et triste, éclairée par deux grands yeux clairs d'une couleur indéfinissable, à la fois verts et violacés comme l'eau d'un étang mort, car c'est à ces yeux que ma curiosité était d'abord allée ; une longue moustache blonde barrait son charmant visage et pourtant ses cheveux frisés étaient noirs. Sir Thomas Welcôme avait la peau très blanche et des mains énormes, d'énormes mains de bourreau, soignées, poncées et, comme les mains d'Ethal, fleuries de bagues à tous les doigts ; il y avait dans ce corps robuste comme une infinie lassitude, on ne sait quelle pesante contrainte. Le regard était mélancolique. Sir Thomas Welcôme répondait à peine aux effusions d'Ethal et semblait être venu à regret (Lorrain, 1901 : 188-189).

23 À comparaison de l'attitude d'Ethal, la distance de Welcôme lui confère l'autorité d'un médiateur puissant. En outre, le désir d'Ethal envers Welcôme désigne l'Irlandais à Fréneuse comme le médiateur du médiateur. La force de Monsieur de Phocas réside dans les affinités inquiétantes de personnages opposés, illustrant les différentes potentialités du héros désirant. Les doigts chargés de bagues, les mains de meurtrier, sont des traits communs à Fréneuse, Ethal et Welcôme. Par sa mélancolie, l'Irlandais ressemble à Fréneuse ; par sa musculature, il évoque Ethal. Quand ce dernier offre à Fréneuse la statue d'Astarté au sexe figurant une tête de mort qui justifie le sous-titre du roman, il précise que c'est devant cette idole qu'a été tué $\mathrm{M}$. de Burdhes - et que Welcôme est suspecté de ce meurtre auquel il doit sa fortune. Non sans ironie, Ethal déclare que la petite idole sera " un chaînon de plus dans l'invisible et forte chaîne qui [les] unit tous les trois » (Lorrain, 1901 : 332). Ethal désigne explicitement l'objet de son culte à Fréneuse. La « Merveille de Leyde ", à ses yeux, est la mort elle-même :

"Ma déesse à moi ", ricanait-il, demi-caressant et sournois. "La mienne est vêtue de la défroque des siècles, mais aucune tête de mort ne grimace sous sa robe : c'est la Mort elle-même, la Mort avec son fard et la transparence de ses décompositions » (Lorrain, 1901 : 278).

Ethal lève le voile sur l'essence vide du désir, sur sa tension vers la mort. À première vue, son idolâtrie pour la « Merveille de Leyde » exprime l'antithèse du credo de Welcôme :

Tout le secret du bonheur humain est là : aimer avec ferveur, s'intéresser passionnément aux choses, rencontrer Dieu partout et l'aimer éperdument dans chaque rencontre, désirer amoureusement toute la nature, les êtres et les choses sans s'arrêter même à la possession, s'user dans le désir effréné du monde extérieur sans même s'inquiéter si le désir est bon ou mauvais. Car toute sensation est une présence, et la splendeur des choses ne vient que de l'ardeur que nous avons pour elles. L'importance est dans le regard et non dans la chose regardée. Qu'importe d'où vienne l'extase, si l'extase nous vient? Toutes les émotions sont comme autant de portes ouvertes vers un prestigieux avenir : le devenir, voilà la religion. Les choses du passé sont déjà mortes ; pourquoi s'attarder sur un cadavre ? Chaque chose possédée est déjà une pourriture, et quand nous regrettons une chose, c'est déjà un germe de mort que nous portons en nous. S'enrichir de désirs, toute la ferveur est là, et la ferveur est une délicieuse usure d'amour. Et Bénarès, depuis des siècles et des siècles, agonise et se meurt dans une ferveur intense, et c'est cette ferveur même, cet extatisme halluciné de toute l'Inde qui la fait vivre et la soutient (Lorrain, 1901 : 338-339).

«Le sujet désirant n'étreint jamais que du vide lorsqu'il s'empare de l'objet » (Girard, 2010 : 191). Si cette maxime est illustrée par Ethal dont la poupée est «la mort elle- 
même ", la quête proposée par Welcôme repose également sur la vanité de l'objet désiré, " cadavre » dès qu'il est possédé, et souligne l'identité entre le désir et la mort comme « délicieuse usure d'amour ». La divinisation du héros par la permanence du désir sans cesse renouvelé est « une permanence dans le néant. Le désir, en effet, n'atteint jamais son véritable objet : il conduit à l'oubli, à la déchéance et à la mort » (Girard, $2010: 269$ ). Rien d'anodin à ce que par-delà les différences, les deux médiateurs, dans le chapitre « Le Piège ", conseillent à Fréneuse de se rendre au musée Gustave Moreau pour y trouver matérialisés les désirs qu'ils lui suggèrent. Ethal lui dit qu'il y trouvera les yeux qu'il cherche; Welcôme lui désigne la toile Le Triomphe d'Alexandre comme un tableau de l'exultation permanente de Bénarès. Le désir de mort communiqué par Ethal et l'agonie désirante érigée par Welcôme en voie de salut aboutissent au même terme : désigner la vanité de l'objet désiré. L'anglais à la laideur dérangeante et l'irlandais à la beauté fascinante sont l'envers et l'avers d'une même médaille, le néant du désir comme désir de néant.

\section{III - Désir de mort et sanctification}

Le désir violent pour l'objet immatériel finit par se cristalliser (cf. Stendhal, 1822) autour de ce qui est insaisissable par définition, la mort elle-même. "La vérité du désir métaphysique ", écrit Girard, " est la mort » (Girard, 2010 : 316) Ce troisième mouvement de notre réflexion demande à lire l'héritage baudelairien de Huysmans et Jean Lorrain à la lumière de la théorie mimétique. En effet, des Esseintes et Fréneuse, par leur fascination pour la violence, sont pris dans le cercle de l'Hérautontimorouménos baudelairien. Ils sont « et la victime et le bourreau » (Baudelaire, 1954 : 150). Comment le système de pensée girardien permet-il d'éclairer cette dualité? L'œuvre d'art ultime prisée par des Esseintes, et de façon plus flagrante par Fréneuse, représente l'imminence de la mort. "Je comprenais enfin la beauté du meurtre, le fard suprême de l'épouvante, l'ineffable empire des yeux qui vont mourir» (Lorrain, 1901: 338), écrit le duc de Fréneuse. Le héros fin-de-siècle ne se fait aucune illusion sur sa bonté. Le «seul contre tous » du héros romantique s'abolit dès que la contemplation de la violence mimétique intègre le héros à la foule. Fréneuse écrit, dès le début de son journal :

Moi-même, qui ai la répugnance et l'horreur de tous les bas instincts, ne suis-je pas instinctivement violent et ordurier, meurtrier et sensuel comme cette foule sensuelle et meurtrière, la foule des émeutes qui jette les sergents de ville à la Seine et criait, il y a cent ans : "Les aristos à la lanterne ! " comme elle vocifère aujourd'hui : « À bas l'armée !» ou : « À mort les Juifs ! » (Lorrain, 1901 : 19)

Le récit autocentré se charge d'allusions à l'affaire Dreyfus, à la Commune, à la défaite de Sedan et à 1792. Fréneuse écrit: "Il y a en moi un fond de cruauté qui m'effraie » (Lorrain, 1901: 36). La plasticité de la violence mimétique, opposée à l'individualité héroïque, bat en brèche l'illusion romantique de l'intégrité.

Le héros fin-de-siècle souffre et fait souffrir. La cruauté, cruor, sang versé, n'a rien de gratuit dans le système girardien. Plus un geste violent semble immotivé, plus sa justification est d'ordre mythique et sacrificiel. L'épisode de la décollation de saint JeanBaptiste à la demande de Salomé porte sens dans les deux romans. Huysmans l'introduit par l'ekphrasis des tableaux de Moreau, Lorrain compare la danseuse Izé Kranile à Salomé. Tous deux font de Salomé une médiatrice dans la triangulation du désir aboutissant à la décollation de saint Jean-Baptiste telle que l'analyse Girard dans Le Bouc émissaire au 
chapitre XI. Telle que la décrit Huysmans et telle que la voit des Esseintes, Salomé acquiert le caractère allégorique que lui attribue également Girard.

le se fait l'incarnation contagieuse du désir,

la déité symbolique de l'indestructible Luxure, la déesse de l'immortelle Hystérie, la Beauté maudite, élue entre toutes par la catalepsie qui lui raidit les chairs et lui durcit les muscles ; la Bête monstrueuse, indifférente, irresponsable, insensible, empoisonnant, de même que l'Hélène antique, tout ce qui l'approche, tout ce qui la voit, tout ce qu'elle touche (Huysmans, 1922: 71).

Le motif de la contamination se superpose à la hantise de l'épidémie, présente tant dans $A$ rebours que dans Monsieur de Phocas, et fait de la danseuse, comme d'Hélène de Troie, celle " par qui le scandale arrive ». Décrivant L'Apparition de Moreau, Huysmans insiste sur le regard accusateur de la tête coupée, à travers lequel le prophète accuse la danseuse et la désigne à la mise à mort figurée dans les Actes de Pilate que commente René Girard. Centre des regards, elle est marquée pour la mort.

31 L'analogie entre le poète et le prophète mérite d'être nuancée. Des Esseintes comme Fréneuse sont plus proches du vieux roi saisi de désir que du prophète contempteur des matérialités charnelles. Jean Lorrain, à travers le personnage d'Izé Kranile, présente l'effet d'une Salomé contemporaine et éternelle sur les sens éteints de Fréneuse (Lorrain, 1901 : 50). Le héros fin-de-siècle est fasciné par l'aptitude de la fillette à rendre matériel un désir abstrait. Le désir de la mort de saint Jean Baptiste se change en désir d'un objet d'art maniable, possédable, une tête sur un plat ; la mort comme œuvre plastique.

Cependant, cette tête coupée a un sens triple. C'est la tête de saint Jean-Baptiste, qui, comme médiateur, a donné aux premiers disciples le désir de suivre Jésus en le désignant comme « l'Agneau de Dieu ${ }^{4} »$; c'est la tête d'Orphée, qui fascine autant des Esseintes que Fréneuse et qui manifeste l'immortalité de la parole poétique ; c'est la tête politique, la tête de Louis XVI et celle des dames de la cour ${ }^{5}$. En définitive, l'objet ultime du désir, c'est la mort de l'illusion romantique du personnage, la fin de sa prétention au titre de prophète, de poète et de roi. À rebours est une double-mort. D'abord, des Esseintes meurt au monde en se retirant dans la thébaïde; ensuite, il meurt à son rêve en retournant à l'anonymat, au silence et à l'oubli :

Dans deux jours je serai à Paris; allons, fit-il, tout est bien fini; comme un raz de marée, les vagues de la médiocrité humaine montent jusqu'au ciel et elles vont engloutir le refuge dont j'ouvre, malgré moi, les digues. Ah! le courage me fait défaut et le cœur me lève ! (Huysmans, $1922: 290$ ).

Outre les souteneurs, les journalistes et les mondains, Fréneuse a deux médiateurs, Ethal qui empoisonne ses modèles et Welcôme suspecté d'avoir étranglé son bienfaiteur ; mais le duc est conscient, dix ans avant de les rencontrer, que ses propres mains sont celles d'un étrangleur (Lorrain, 1901 : 23 ; 360). La confusion entre Fréneuse et Ethal est telle que les policiers et les journalistes croient au suicide lorsqu'ils trouvent le cadavre empoisonné du peintre empoisonneur. Presque en même temps qu'Ethal, le duc de Fréneuse meurt et renaît sous le nom de monsieur de Phocas, quand il rencontre enfin un médiateur externe, la voix qui le pousse vers l'Orient.

34 En définitive, ce qui meurt à la fin de ces deux romans est la vanité du héros. La conversion de Huysmans incite à voir en À rebours les prémices d'En route (1895), ce que confirme l'oraison jaculatoire des dernières lignes : 
Seigneur, prenez pitié du chrétien qui doute, de l'incrédule qui voudrait croire, du forçat de la vie qui s'embarque seul, dans la nuit, sous un firmament que n'éclairent plus les consolants fanaux du vieil espoir ! (Huysmans, $1922: 290$ ) demande à être vu non dans sa force mais dans sa vulnérabilité, non dans ce qu'il a mais dans ce qui lui fait défaut. Cette pitié dont René Girard fait le trait essentiel de Jésus est la seule issue hors de l'enfer du désir mimétique. La bienveillance qu'elle manifeste s'oppose symétriquement à l'irrésistible pulsion cruelle dont sont saisis des Esseintes et Fréneuse. Elle conduit à regarder et à être regardé non dans les qualités positives, imitées et imitables, mais dans la lacune, la faiblesse, le manque. C'est la vulnérabilité du personnage qui lui confère densité et unicité. Le regard de compassion détruit la distance qui fait du héros un médiateur admirable et imitable, désamorçant ainsi la dispersion engendrée par la rivalité mimétique. La structure cyclique d'À rebours et de Monsieur de Phocas renvoie la fin de la narration à son origine de la même manière que le héros est renvoyé à un néant. Déparés de leur tentation romantique d'être unique, des Esseintes et Fréneuse ont pour dernier mouvement un retour sur soi, une prise de conscience de leur néant essentiel comme êtres de désir. C'est, dans les deux cas, une conversion, au sens où René Girard peut écrire: "Toutes les conclusions romanesques sont des conversions.» (Girard, $2010: 330$ )

38 La théorie du désir mimétique élaborée par René Girard permet de mieux appréhender les éléments disparates qui constituent le type du héros fin-de-siècle. Amateur de beauté, il désire la détruire ; épris de solitude, il déplore de n'avoir personne avec qui partager sa faim d'idéal. En définitive, ces stylites de l'unicité révèlent la vanité du désir. Du fond de leur enfermement, ils manifestent la crise propre à leur temps, la banqueroute de l'épopée. La volonté d'être unique, poussée à la pathologie ou au ridicule, fait de Des Esseintes et de Fréneuse les derniers dandys romantiques. Pourtant, Huysmans et Jean Lorrain les donnent volontairement à lire comme des palimpsestes romanesques, des 
foyers de résonance esthétique où vient se répercuter une généalogie de modèles, Baudelaire en tête. Le désir d'être unique semble un symptôme d'incurable romantisme grevant la fin de siècle, mais, tissé au constant appel au regard voire à la pitié du lecteur comme juge, ce romantisme s'inverse en romanesque. De sa décomposition-même, en sacrifiant l'indépendance et l'unicité héroïques de son personnage principal, le roman fin-de-siècle ouvre une brèche hors de la crise du modèle épique.

\section{BIBLIOGRAPHIE}

BAUDELAIRE, Charles (1868). Curiosités esthétiques, in Euvres complètes, 2 vol. Paris : Michel Lévy.

BAUDELAIRE, Charles (1885). L'Art romantique. [1852], Paris : Calmann Lévy.

BAUdelaire, Charles (1954). CEuvres complètes. Paris : Gallimard, « La Pléiade ».

BAUdelaire, Charles (1908). CEuvres posthumes. Paris : Mercure de France.

GENETTE, Gérard (1982). Palimpsestes. Paris : Éditions du Seuil.

GIRARD, René (2010). Mensonge romantique et vérité romanesque. Paris : Fayard, « Pluriel » [Paris,

Grasset, 1961.]

GIRARD, René (1982). Le Bouc émissaire. Paris : Grasset et Fasquelle, « Le Livre de poche - Essais ».

GIRARD, René (1999). Je vois Satan tomber comme l'éclair. Paris : Grasset et Fasquelle, « Le Livre de

poche - Essais ».

GIRARD, René (2008). Anorexie et désir mimétique. Paris : l'Herne, « Carnets ».

HUYSMANS, Joris-Karl (1922). À rebours. Paris : Georges Crès. [(1884). Paris : Charpentier.]

HUYSMANS, Joris-Karl « Le Nouvel album d'Odilon Redon », La Revue indépendante, février 1885, pp. 291-296.

KISTEMAEKERS, Henry (1897). «L'Heure du sang » in Jean-David Jumeau-Lafond (2001) Naissance du fantôme. Paris : La Bibliothèque.

LORRAIN, Jean (2007). « Les Yeux », in Chroniques d'art, éd. Thalie Rapetti, Paris : Champion, pp. 335-339.

LORRAIN, Jean (1901). Monsieur de Phocas - Astarté. Paris : Ollendorff.

MAUPASSANT, Guy de (1884). «Par-delà ». Gil Blas, 10 juin 1884, p. 1.

MATZNEFF, Gabriel (1994). Le Taureau de Phalaris. Paris : La Table Ronde.

STENDHAL (1822). De l'amour. Paris : Librairie universelle.

VELLAY, Charles. « Contes au bord de la mer. » Mercure de France, t. XXII, n 90, juin 1897, pp. 473-480. 


\section{NOTES}

1. Il eût été intéressant de mettre en regard les analyses que fait René Girard de l'anorexie (Girard, 2008) et les détails alimentaires dont sont émaillés À rebours et Monsieur de Phocas.

2. Madeleine Deslandes (1866-1929), femme de lettres, a publié sous les pseudonymes d'Ossit et Ilsée. Jean Lorrain la fait figurer dans Monsieur de Phocas sous le pseudonyme de la baronne Desrodes.

3. «Et Thomas Welcôme qui n'arrive pas, grognait Claudius en consultant sa montre. » (Lorrain, $1901: 175$ « Et Welcôme qui ne vient pas. » (Lorrain, $1901: 177)$

4. Jean $1: 29$ et $1: 36$.

5. Willie Stephenson fascine Fréneuse par son air de «beauté d'échafaud» (Lorrain, 1901: 42) : «C'était une beauté d'échafaud dont la fragilité même appelait le viol et la violence, beauté meurtrie qui éveillait en moi des instincts meurtriers. Auprès d'elle, que de fois j'ai songé aux exsangues et douces figures, douces et pourtant impertinentes, des victimes de la Révolution, à ces jolies et longues aristocrates, que les Carrier et les Fouquier-Tinville envoyaient, encore toutes pantelantes de leur luxure, à la noyade ou à la guillotine. » (Lorrain, $1901: 42$ )

\section{RÉSUMÉS}

Dans Mensonge romantique et vérité romanesque, René Girard trace une ligne de démarcation entre les œuvres qui démasquent l'illusion d'un désir individualisé et celles où est entretenu le mensonge romantique. Aux lendemains de la défaite de Sedan, Joris-Karl Huysmans dans À rebours (1884) et Jean Lorrain dans Monsieur de Phocas (1901) associent la dimension médicale du naturalisme au symbolisme préfiguré par Baudelaire. Les personnages centraux respectifs de ces romans, Jean des Esseintes et Jean de Fréneuse, manifestent par leur goût du beau, du rare et de l'étrange une volonté d'être unique qui fut au cœur de l'illusion romantique du début du siècle. Huysmans et Lorrain, en créant des personnages isolés qui revendiquent leur radicale différence, ne font que souligner l'impossibilité de se concevoir comme individualité désirante. Si le modèle du héros est anéanti par la révélation du désir comme néant, il révèle néanmoins la vanité du désir. S'écartant du mensonge romantique, il amorce une sortie hors de la crise du modèle épique.

In Mensonge romantique et vérité romanesque, René Girard draws a line between the novels that reveal the delusion of self-made desires and the ones that nurter this romantic lie. After the defeat of Sedan, Joris-Karl Huysmans in À rebours (1884) and Jean Lorrain in Monsieur de Phocas (1901) combine the medical aspects of naturalism with the symbolism foreshadowed by Baudelaire. The main characters of these novels, Jean des Esseintes and Jean de Fréneuse, cultivate a taste for beauty, rareness and oddity which gives away their romantic desire of being unique. These self-isolated and willingly lonely characters underline the impossibility of conceiving onsefelf as a desiring individual. As he discovers the emptiness of his desire, the character fails at becoming a hero, and yet brings to light the vanity of desire. As he steps aside from the romantic lie, he opens a path out of the crisis of the epic.

31 janvier 2018 
INDEX

Mots-clés : fin-de-siècle, dandysme, crise du modèle épique, Lorrain (Jean), Huysmans (JorisKarl)

Keywords : fin-de-siècle, dandysm, crisis of the epic, Lorrain (Jean), Huysmans (Joris-Karl)

\section{AUTEUR}

\section{MARIE KAWTHAR DAOUDA}

Université de Paris-IV Sorbonne

marie.k.daouda[at]gmail.com 